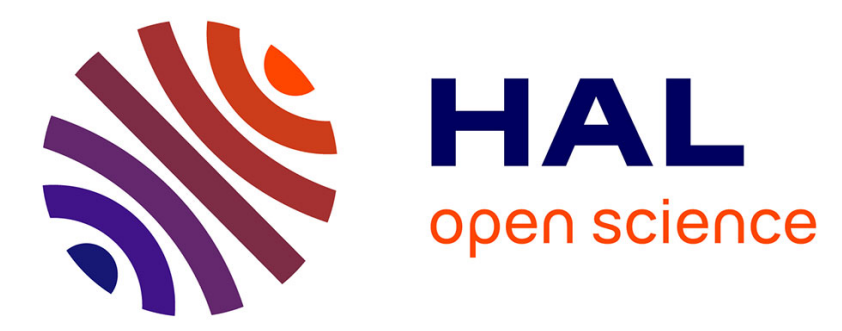

\title{
Systèmes de culture et statut organique des sols dans le Noyonnais : application du modèle de Hénin-Dupuis
}

\author{
Jean Boiffin, Jules Kéli Zagbahi, Michel Sebillotte
}

\section{To cite this version:}

Jean Boiffin, Jules Kéli Zagbahi, Michel Sebillotte. Systèmes de culture et statut organique des sols dans le Noyonnais: application du modèle de Hénin-Dupuis. Agronomie, 1986, 6 (5), pp.437-446. hal-00884894

\section{HAL Id: hal-00884894 \\ https://hal.science/hal-00884894}

Submitted on 1 Jan 1986

HAL is a multi-disciplinary open access archive for the deposit and dissemination of scientific research documents, whether they are published or not. The documents may come from teaching and research institutions in France or abroad, or from public or private research centers.
L'archive ouverte pluridisciplinaire HAL, est destinée au dépôt et à la diffusion de documents scientifiques de niveau recherche, publiés ou non, émanant des établissements d'enseignement et de recherche français ou étrangers, des laboratoires publics ou privés. 


\section{Systèmes de culture et statut organique des sols dans le Noyonnais : application du modèle de Hénin-Dupuis}

Jean BOIFFIN, Jules KÉLI ZAGBAHI (1) \& Michel SEBILLOTTE

I.N.A.P.G., Chaire d'Agronomie, 16, rue Claude-Bernard, F 75005 Paris

RÉSUMÉ

On cherche à tester le modèle de Hénin-Dupuis et l'ensemble des références numériques qui lui sont associées à partir d'une enquête sur des parcelles supposées au voisinage de l'équilibre organique et correspondant à une large gamme de sols et de systèmes de culture. Pour cela, on confronte les teneurs d'équilibre calculées à partir des informations sur le sol et le régime d'entretien organique aux teneurs observées. Les deux séries de valeurs ainsi obtenues sont corrélées mais les valeurs calculées sont, en moyenne, inférieures aux valeurs observées surtout si elles sont exprimées en carbone plutôt qu'en matière organique. Différentes hypothèses, susceptibles de rendre compte de ce biais systématique et de la dispersion résiduelle autour de la régression, sont discutées. Vis-à-vis du biais, l'hypothèse la plus plausible est celle d'une surestimation de la minéralisation ; quant à la dispersion, elle est probablement encore liée à celle des statuts organiques antérieurs. En l'état actuel, le modèle de HÉNIN-DuPUIS peut être considéré comme un bon instrument de classement des systèmes de culture vis-à-vis de leurs conséquences sur l'évolution de l'état organique du sol dans une région donnée, mais, par rapport à la description d'une évolution, son caractère monocompartimental est sans doute trop simpliste.

Mots clés additionnels : Matière organique, résidus de culture, fumier.

We tested the HÉNIN-Dupuis model and the numerical references associated with it. This was done by means of a survey on 89 farm plots, supposed to be close to organic equilibrium and corresponding to a wide range of soils (fig. 1) and cropping systems in a restricted area. For each plot, the expected value of organic content (calculated from information collected on soil and on organic supply) was compared with the observed content. Expected and observed values proved to be correlated (fig. 2 and 3), but expected ones were systematically lower than those observed, especially if the content was expressed as carbon instead of organic matter. Different hypotheses were examined to explain this bias and the residual dispersion around the linear regression (expected content versus observed). It was concluded that the most plausible origins were, for the bias, an overestimation of the annual rate of mineralization $\mathrm{k}_{2}$, and, for the residual dispersion, the influence of unknown former organic status. In addition, the collection of numerous results concerning plots where farmyard manure was used allowed us to estimate the isohumic coefficient $\left(\mathrm{k}_{1}\right)$ of this product $(0.34)$. In the present state of knowledge, the HÉNIN-DupuIs model with associated numerical references can be considered as a good tool for grading cropping systems from the point of view of organic management. However in order to predict the change in organic content of a given soil with time, this tool seems inadequate, due to its monocompartmental character.

Additional key words : Organic matter, plant residues, farmyard manure.

\section{INTRODUCTION}

Le diagnostic et la prescription concernant l'entretien organique d'un sol doivent s'appuyer sur des moyens de prévision de l'évolution de son statut orga-

(1) Adresse actuelle : I.R.C.A., Bimbresso, 01 BP 1536 Abidjan 01 Côte-d'Ivoire. nique. Ce problème est lié à celui de la caractérisation des matières organiques du sol. Or on ne dispose pas à l'heure actuelle d'une méthode de fractionnement qui soit à la fois praticable sur une large échelle et reconnue pertinente vis-à-vis de l'ensemble des propriétés agronomiques concernées : les fractionnements de type densimétrique (HÉNIN \& TURC, 1949 ; HÉNIN et al., 1959 ; MONNIER et al., 1962) ne résolvent que 
certains aspects de la question. Pour le moment, la notion de statut organique se réduit dans la pratique à sa plus simple expression : la teneur en matière organique totale, le plus souvent déduite d'un dosage du carbone.

Bien que trop global, ce paramètre mérite cependant d'être pris en compte dans la prévision de nombreux critères participant au diagnostic de la fertilité : capacité d'échange (MYERS, 1937 ; RUELLAN \& DELETANG, 1967) ; stabilité structurale (MONNIER, 1965 ; RÉMY, 1971 ; BOIFFIN \& FLEURY, 1974) ; humidités caractéristiques des changements de consistance de la terre (RÉMY, 1971 ; GUÉRIF \& FAURE, 1979) ; aptitude à la fissuration (OSTY, 1971a) ; humidité équivalente (OSTY, 1971b) ; mobilité de l'eau non saturante (MORIZET \& MILlET, 1971) ; quantité d'azote minéralisée (DELPHIN \& TIQUET, 1986).

La prévision de l'évolution fait donc essentiellement appel aux modèles monocompartimentaux, dont une formulation classique est celle de HÉNIN-DUPUIS (1945). C'est en référence à ce modèle qu'ont été acquises les données numériques vulgarisées ces dernières années et permettant, dans une situation culturale donnée, de prévoir les sens d'évolution des teneurs et les valeurs d'équilibre (JACQUIN, 1963 ; MONNIER, 1966 ; RÉMY \& MARIN-LAFLĖCHE, 1976 ; RÉMY et al., 1975 ; MULLER, 1982).

Or ce modèle et les références qui lui sont associées ne reposent pas sur un très grand nombre de résultats expérimentaux et n'ont, à notre connaissance, jamais été à proprement parler testés. Ce test peut être entrepris de 2 manières :

- confrontation entre des évolutions de teneurs respectivement prévues par le modèle et observées. Cette voie nécessite une longue période d'observation avec un bon contrôle des apports et une situation témoin sans apport ; elle ne peut donc être mise en œuvre que sur un très petit nombre de sites expérimentaux qui sont, en fait, précisément ceux déjà utilisés pour établir, voire préciser, les valeurs numériques des coefficients du modèle (par exemple : MOREL, 1968 ; DELPHIN \& CONESA, 1979) ;

- confrontation des teneurs d'équilibre prévues et observées. A supposer que l'on puisse identifier et caractériser des parcelles effectivement proches de l'équilibre organique, cette approche peut se réaliser à un moment donné et sans connaître les statuts organiques antérieurs de ces parcelles. La difficulté majeure réside dans l'incertitude qui pèse nécessairement sur des statuts organiques très anciens et néanmoins encore influents compte tenu de la lenteur des évolutions.

Nous avons tenté de mettre en œuvre cette $2^{\mathrm{e}}$ démarche dans le Noyonnais, petite région située au NordOuest de l'Oise, où la variété tant des systèmes de production rencontrés (AUBRY et al., 1986) que les sols (BEGON et al., 1976) nous donnait l'occasion de tester le modèle et les références numériques associées pour une large gamme de variation de chacun des paramètres du modèle. Ce faisant, nous espérions aussi que les caractéristiques de la distribution des écarts entre prévisions et observations pourraient nous renseigner sur l'origine et les voies de rectification d'éventuelles défaillances du système de prévision (KÉLI, 1984).

\section{MÉTHODES D'ENQUÊTE}

\section{A. Choix des situations observées}

Pour obtenir une gamme de variation importante des régimes d'entretien organique, on a tout d'abord recherché des exploitations ayant des systèmes de production contrastés (présence ou absence d'élevage, degré d'intensification de celui-ci, vente ou achat de paille et de fumier, fréquence de la pomme de terre dans la succession des cultures...). Dans un $2^{\mathrm{e}}$ temps, au sein de ces exploitations, on a choisi des parcelles répondant aux conditions suivantes :

- précision suffisante des informations concernant les régimes d'entretien organique au cours des 10 dernières années (cette période varie en fait de 5 à 25 ans, selon les exploitations) ;

- certitude, de la part de l'agriculteur, que le système de culture n'a pas subi de modifications importantes dans les 25 dernières années et que la parcelle n'a pas porté de prairie de longue durée depuis au moins 50 ans. $A$ contrario, et pour vérifier le rôle effectif de cette règle de sélection, quelques parcelles où le retournement de prairie permanente était plus récent et précisément situé dans le temps, ont été étudiées ;

- absence de forte hétérogénéité ou de caractères de terrain très particuliers par rapport au domaine courant d'établissement des normes associées au modèle de HÉNIN-DuPUIS (hydromorphie très marquée, forte pente...). Par contre, si des zones de textures différentes importantes en surface se présentaient au sein d'une parcelle, elles ont pu être étudiées séparément ;

- présence, lors de la période d'enquête, d'une céréale d'hiver. Les prélèvements et mesures tyant tous été réalisés au printemps, cette procédure réduit la variabilité liée, d'une part, à l'évolution intraannuelle des résidus de culture, (dont GUÉRIF (1982) a souligné l'importance), d'autre part, à l'état structural de la couche arable.

Au total, 98 zones incluses dans 85 parcelles supposées à l'équilibre ou à son voisinage, appartenant à 42 exploitations, ont été étudiées. Elles correspondent à une très large gamme de textures (fig. 1). En outre, un grand nombre de ces sols sont faiblement calcaires.

\section{B. Observations et contrôles réalisés}

1) Dans chaque zone retenue au sein d'une parcelle choisie, 2 ou 3 stations (surface $=100 \mathrm{~m}^{2}$ environ) ont été étudiées. Sur chaque station sont déterminées :

- la (ou les) profondeur(s) de labour (moyenne de 10 mesures sur $2 \mathrm{~m}$ de large, entre la surface et chaque fond identifié),

- la densité apparente de la couche travaillée (moyenne de 5 mesures à l'aide d'un cylindre enfoncé verticalement jusqu'au fond de labour),

- les caractéristiques analytiques classiques ( 5 fractions granulométriques, carbone organique par la méthode ANNE, calcaire total) sur un échantillon constitué à partir de 15 carottes prélevées jusqu'au 


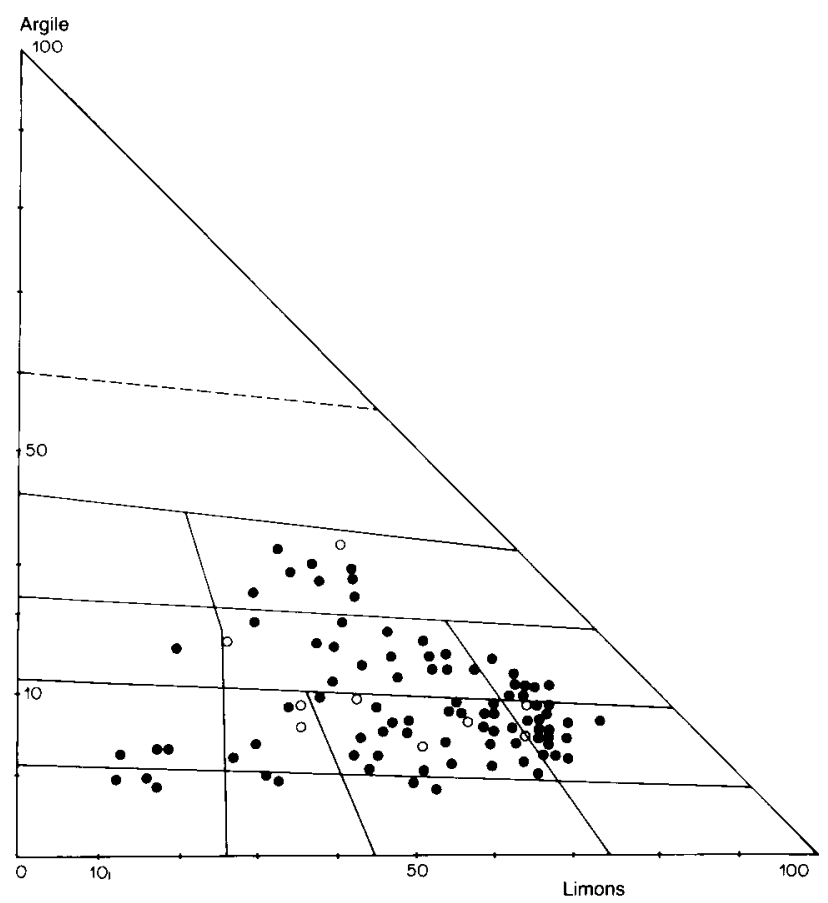

Figure 1

Texture des échantillons étudiés.

0 : Retournement récent de prairie $(\leqslant 16$ ans).

- : Terres labourées depuis très longtemps ( $\geqslant 50$ ans).

$0:$ Recent conversion from pasture to cultivation.

- : Cultivation for a very long time.

fond de labour le plus profond de la succession actuelle.

Les intervalles de confiance des principales déterminations concernant chaque zone sont, en moyenne, et à $\mathbf{P}=0,05$, de $\pm 0,6 \mathrm{~cm}$ pour la profondeur de labour, $\pm 0,03$ pour la densité apparente et $\pm 0,7$ p. 1000 pour la teneur en matière organique.

2) Auprès de l'agriculteur, ont été enregistrés pour chaque parcelle :

- la succession des cultures pratiquées pendant la période connue avec précision,

- les rendements (le plus souvent connus en moyenne au niveau de l'exploitation),

- le devenir des résidus de récolte,

- les apports d'amendements organiques.

\section{CALCUL DES TENEURS D'ÉQUILIBRE}

\section{A. Principe de calcul}

D'après le modèle de HÉNIN-DuPUIS, la teneur d'équilibre obtenue sur une parcelle donnée lorsqu'elle est indéfiniment soumise au même régime d'apports, peut s'écrire :

$$
\tau_{\mathrm{e}}=\frac{\mathrm{k}_{1} \mathrm{~m}}{\mathrm{k}_{2} \mathrm{M}}
$$

où : $\mathrm{k}_{1} \mathrm{~m}$ représente l'apport moyen annuel au stock organique du sol (m étant l'apport moyen annuel de matière organique brute, $\mathrm{k}_{1}$ le rendement global en matière organique du sol de cet apport), $\mathrm{k}_{2}$ est le coefficient de minéralisation annuel, supposé dépendre principalement de la constitution minérale du sol et du climat,

$M$ est la masse de terre fine sèche contenue sur un hectare entre la surface et le fond du labour le plus profond de la succession.

En fait, le terme moyen $\mathrm{k}_{1} \mathrm{~m}$ est la résultante des contributions de diverses sources d'apports; il faudrait écrire :

$$
\tau_{e}=\frac{\sum_{i}\left(k_{1 i} m_{i} f_{i}+k_{1 j}^{\prime} m_{i}^{\prime} f_{i}^{\prime}\right)+\sum_{j} k_{1 j} m_{j} f_{j}}{k_{2} M}
$$

où :

$\mathrm{k}_{\mathrm{li}}, \mathrm{k}_{\mathrm{li}}^{\prime}, \mathrm{k}_{\mathrm{lj}}$ sont les coefficients de transformation en matière organique du sol des apports effectués respectivement par les restitutions obligatoires (racines et chaumes pour les céréales, par exemple), par les restitutions facultatives (parties aériennes), par les amendements organiques,

$\mathrm{m}_{\mathrm{i}}, \mathrm{m}_{\mathrm{i}}^{\prime}, \mathrm{m}_{\mathrm{j}}$ sont les masses correspondant à ces 3 types de restitutions,

$f_{i}, f_{i}^{\prime}, f_{j}$ leurs fréquences de réalisation au sein de la succession.

\section{B. Choix d'un mode d'expression}

Les variations des teneurs du sol, les apports et les pertes peuvent être exprimés soit en matière organique, soit en carbone.

Dans le $1^{\text {er }}$ cas, il faut convertir les teneurs en carbone du sol en matière organique, en utilisant le coefficient 1,72 traditionnel. Celui-ci apparaît, en fait, assez discutable, certains spécialistes estimant qu'un coefficient 2 correspond mieux aux teneurs réelles en carbone des matières organiques des sols (BROADBENT, 1953 ; MONNIER, RÉMY, comm. pers.).

Dans le $2^{e}$ cas, il faut convertir les masses de matière sèche des apports en carbone. Les références disponibles sur les teneurs en carbone des matières végétales semblent suffisamment nombreuses et convergentes pour que cette opération n'introduise pas par elle-même d'erreur importante.

Le choix de l'une ou l'autre de ces modalités d'expression retentit sur les valeurs du coefficient $k_{1}$. En effet, si on suppose que $k_{1}$ est déduit d'une variation $(\Delta \mathrm{MO}$ ou $\Delta \mathrm{C})$ du stock organique du sol consécutive à un apport moyen annuel $m$ renouvelé $n$ années et dont la teneur en carbone est $c$, on a, en $1^{\text {re }}$ approche :

$\mathrm{k}_{1}(\mathrm{mo})=\frac{\Delta \mathrm{MO}+\mathrm{nk}_{2} \mathrm{MO}}{\mathrm{n} \cdot \mathrm{m}}$ et $\mathrm{k}_{1}(\mathrm{c})=\frac{\Delta \mathrm{C}+\mathrm{nk}_{2} \mathrm{C}}{\mathrm{n} \cdot \mathrm{m} \cdot \mathrm{c}}$

Si MO est déduit de $\mathrm{C}(\mathrm{MO}=1,72 \mathrm{C})$, il vient :

$$
\mathrm{k}_{1}(\mathrm{mo})=1,72 \cdot \mathrm{c} \cdot \mathrm{k}_{1}(\mathrm{c})
$$

et, comme c est de l'ordre de 0,40 , on a :

$$
\mathrm{k}_{1}(\mathrm{mo}) \simeq 0,7 \mathrm{k}_{1} \text { (c) }
$$


Toute valeur numérique de $\mathrm{k}_{1}$ devrait donc être assortie du mode d'expression des teneurs choisi. Malheureusement, cette précision fait souvent défaut et il faut se reporter aux travaux expérimentaux originels : ainsi les valeurs déterminées pour la paille par MONNIER (1965) et pour différents produits par MULLER (1966) et par DELAS \& MOLOT (1983) se réfèrent explicitement au carbone. Mais l'ambiguïté subsiste malgré tout pour un grand nombre de valeurs.

Devant cette situation, nous avons préféré effectuer les 2 types de calculs, en appliquant dans chaque cas le même jeu de coefficients $k_{1}$, et discuter les résultats obtenus avec l'une et l'autre des modalités.

Remarquons que cette discussion n'a pas à s'appliquer au coefficient $k_{2}$ dont les déterminations usuelles (diminutions de teneurs en régime d'apport nul) ne font intervenir aucune hypothèse particulière relative à la teneur en carbone (il en irait différemment si l'on voulait, par exemple, déterminer un coefficient de minéralisation à partir d'un dégagement de gaz carbonique).

\section{Estimation des différents termes}

\section{Fréquences (termes $f_{i}, f_{i}, f_{j}$ )}

Elles sont déduites des enregistrements auprès de l'agriculteur, précédemment mentionnés. Il convient de noter que, si le nombre " $\mathrm{N}$ » d'années pour lesquelles les cultures et amendements sont connus est faible, une erreur importante peut être faite en admettant sans précaution :

$$
f_{i(o u j)}=\frac{n_{i(o u j)}}{N}
$$

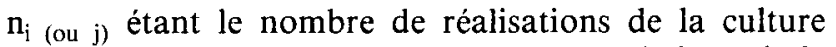
« $\mathrm{i}$ » ou de l'amendement « $\mathrm{j}$ » au cours de la période connue. Ceci est particulièrement à craindre si $\mathbf{n}_{\mathrm{i}(\mathrm{ou}} \mathrm{j}$ ) est lui-même faible. Une vérification systématique de la périodicité des amendements organiques et des cultures a donc été réalisée auprès des agriculteurs.

\section{Masses (termes $m_{i}, m_{i}^{\prime}, m_{j}$ )}

Pour les différentes cultures (indice i), la donnée de base est un rendement récolté (généralement en moyenne de l'exploitation), pour une année donnée. $\mathrm{Ce}$ rendement moyen est affecté à la parcelle. Les restitutions par les parties aériennes et par les racines sont déduites du rendement-récolte en utilisant des coefficients de proportionnalité choisis d'après les différentes sources expérimentales ou bibliographiques (Annexe 1). Les teneurs en carbone retenues correspondent au mode des valeurs citées dans la littérature.

Pour les amendements organiques, la donnée de base est la masse de produits frais que l'agriculteur déclare avoir épandue par hectare.

Les teneurs en matière sèche et en carbone sont fixées conventionnellement pour chaque type d'amendement selon les données de la littérature (Annexe 1).

\section{Coefficients « $k_{1}$ »}

Ils sont également issus de la bibliographie et présentés en Annexe 1.

\section{Coefficients « $k_{2} 》$}

Ils sont déduits de la formule empirique proposée par RÉMY \& MARIN-LAFLÈCHE (1974), dans le programme CERES, soit :

$$
\mathrm{k}_{2}=\frac{\mathrm{K}}{(\mathrm{A}+200)(\mathrm{Ca}+200)}
$$

où $\mathrm{A}$ et $\mathrm{Ca}$ sont les teneurs en argile et calcaire (p. 1000 ) et où le numérateur $K$, traduisant l'influence climatique, doit être ajusté à chaque région.

Ici, on prend initialement $K=1200$, valeur adoptée pour la station agronomique de Laon, peu éloignée de la zone étudiée.

\section{RÉSULTATS ET DISCUSSION}

\section{A. Gammes de variation des paramètres du modèle}

Les régimes d'apport aboutissent à des contributions moyennes annuelles au stock organique du sol $\left(\mathrm{k}_{1} \mathrm{~m}\right.$ moyen annuel) variant de 300 à $1500 \mathrm{~kg}$ en matière organique, 100 à $550 \mathrm{~kg}$ en carbone. Cette variabilité, très importante, est déterminée principalement par celle de la fréquence des apports de fumier. La valeur modale du coefficient $k_{2}$ est de 0,014 , les extrêmes étant respectivement de 0,008 et 0,02 ; ce qui correspond à l'importante variabilité texturale déjà mentionnée.

La variabilité des stocks de terre est également très importante (2 300 à $5600 \mathrm{t} / \mathrm{ha}$, avec un mode peu marqué vers $4200 \mathrm{t} / \mathrm{ha}$ ). Les variations de profondeur de labour ( 25 à $40 \mathrm{~cm}$ ) semblent liées principalemęnt à la teneur en argile : lorsque celle-ci croît, la valeur maximum et la gamme de variation des profondeurs de labour diminuent (KÉLI, 1984). Quant à la densité apparente, elle varie dans l'échantillon de 0,9 à 1,6, elle aussi en fonction de la teneur en argile, les sols les plus argileux étant les moins denses.

\section{B. Confrontation entre les teneurs observées et les teneurs d'équilibre calculées. Résultats d'ensemble}

Cette confrontation est présentée sur les fig. 2 et 3 , avec calculs exprimés respectivement en matière organique et carbone.

Il y a effectivement corrélation entre les teneurs observées et les teneurs calculées. On a les équations de régression suivantes (sans prendre en compte les retournements récents de prairies) :

- en matière organique :

$$
\begin{gathered}
\tau=0,598 \tau_{\mathrm{e}}+8,77 \quad(\mathrm{r}=0,703 \text { pour } 89 \text { couples }) \\
- \text { en carbone : } \\
\begin{array}{c}
\tau=0,921 \tau_{\mathrm{e}}+5,07 \quad(\mathrm{r}=0,717 \text { pour } 89 \text { couples }) \\
\left(\tau \text { et } \tau_{\mathrm{e}} \text { en } \mathrm{p} .1000\right)
\end{array}
\end{gathered}
$$

Par ailleurs, on vérifie que les points expérimentaux correspondant aux retournements récents de prairies 


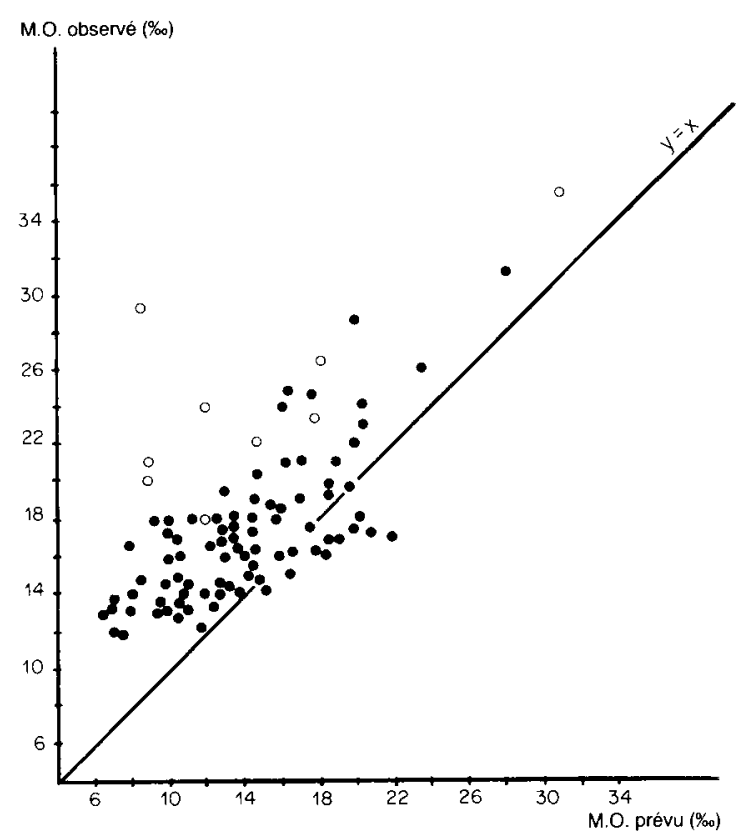

Figure 2

Comparaison des teneurs en matière organique des sols prévues et observées; expression en matière organique.

$O$ : Retournement récent de prairie ( $\leqslant 16$ ans).

- : Terres labourées depuis très longtemps ( $\geqslant 50$ ans).

Expected versus observed soil organic matter contents; content expressed as organic matter.

0 : Recent conversion from pasture to cultivation.

- : Cultivation for a very long time.

(replacés sur les fig. 2 et 3 ) et non pris en compte dans les calculs de régressions sont nettement situés audessus de celles-ci. L'écart moyen de ces points à la régression (tabl. 1) est très significativement différent de zéro.

Il s'avère donc que le classement d'ensemble des situations a été assez correctement prévu à l'aide du modèle de HÉNIN-DUPUIS et des références numériques qui lui sont associées.

Cependant, on peut faire la remarque suivante :

La forme des relations et leur position par rapport

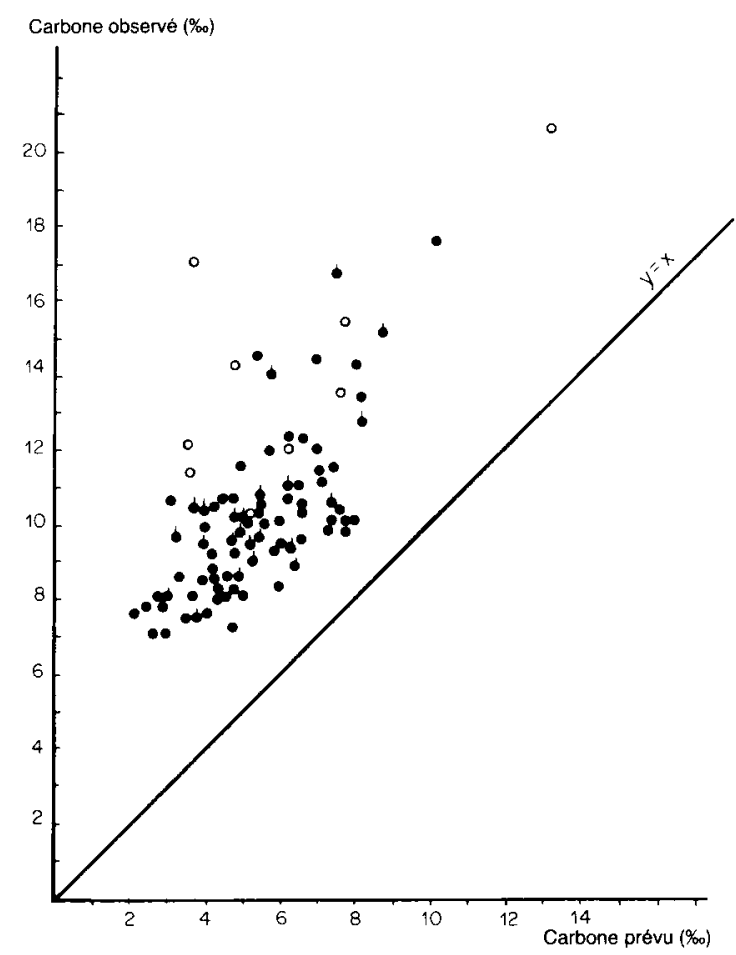

Figure 3

Comparaison des teneurs en matière organique des sols prévues et observées; expression en carbone.

0 : Retournement récent de prairie $(\leqslant 16$ ans).

- : Terres labourées depuis très longtemps ( $\geqslant 50$ ans).

- : Connaissance plus précise des apports.

Expected versus observed soil organic matter contents; content expressed as carbon.

0 : Recent conversion from pasture to cultivation.

- : Cultivation for a very long time.

- More accurate knowledge of organic supply.

à la $1^{\text {re }}$ bissectrice est différente selon qu'on calcule les résultats en carbone ou en matière organique : les pentes sont différentes au seuil de 5 p. 100 et la pente de la régression matière organique est très significativement différente de 1. Il est tout à fait logique que les 2 équations aient une forme différente dans les 2 cas

TABLEAU 1

Analyse des écarts résiduels à la relation (5).

Analysis of residuals for relation (5)

\begin{tabular}{|c|c|c|c|c|c|}
\hline \multicolumn{3}{|c|}{ Type de parcelle considéré } & $\begin{array}{l}\text { Nombre } \\
\text { de cas }\end{array}$ & $\begin{array}{l}\text { Moyenne des écarts } \\
\text { à la régression } \\
(\% / \text { oo de C })\end{array}$ & Observations \\
\hline \multicolumn{3}{|c|}{ Retournements récents de prairies } & 9 & 3,25 & Ecart différent de $O$ à $P \ll 0,01$ \\
\hline \multirow{2}{*}{$\begin{array}{l}\text { Terres labourées } \\
\text { depuis très longtemps }\end{array}$} & 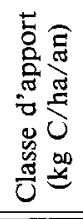 & $\begin{aligned} \mathrm{k}_{1} \mathrm{~m} & <250 \\
250 \leqslant \mathrm{k}_{1} \mathrm{~m} & <350 \\
\mathrm{k}_{1} \mathrm{~m} & \geqslant 350\end{aligned}$ & $\begin{array}{l}37 \\
31\end{array}$ & $\begin{array}{r}0,59 \\
-0,08 \\
-0,37 \\
\end{array}$ & $\begin{array}{l}\text { Effet de la classe } k_{1} m \text { significatif } \\
\text { (à } P \simeq 0,06 \text { ) (Ecart significatif entre } \\
\text { les deux extrêmes) }\end{array}$ \\
\hline & 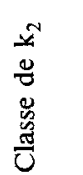 & $\begin{aligned} \mathrm{k}_{2} & <0,014 \\
0,014 \leqslant \mathrm{k}_{2} & <0,016 \\
\mathrm{k}_{2} & \geqslant 0,016\end{aligned}$ & $\begin{array}{l}29 \\
31\end{array}$ & $\begin{array}{r}0,24 \\
-0,12 \\
-0,19\end{array}$ & $\begin{array}{l}\text { Effet de la classe } k_{2} \text { non significatif } \\
(P \gg 0,10)\end{array}$ \\
\hline
\end{tabular}


puisque les coefficients $k_{1}$ pris en compte dans les calculs sont identiques alors qu'ils devraient mathématiquement être différents. Mais il convient de rechercher quel est le cas de figure le plus plausible :

- avec une ampleur différente dans les 2 cas, les valeurs observées sont en général plus élevées que les valeurs calculées ; les 2 ordonnées à l'origine sont très significativement différentes de 0 ;

- dans les 2 cas, une dispersion assez importante subsiste autour de la droite de régression : seulement 50 p. 100 de la variation observée sont expliqués par celle des teneurs prévues. L'examen du tableau 1 montre que la distribution des écarts résiduels est pratiquement indépendante des estimations sur $\mathrm{k}_{2}$ et est, par contre, légèrement dépendante des estimations d'apports $\left(k_{1} m\right)$, mais ceci n'explique qu'environ 5 p. 100 de la viariance résiduelle.

Nous sommer donc conduits à examiner successivement les différentes sources d'écart entre observations et prévisions en considérant, en $1^{\text {re }}$ approximation, les erreurs expérimentales portant sur les mesures de terrain comme secondaires.

\section{Discussion sur le rôle du statut organique antérieur}

\section{Problème du biais systématique}

Nous devons tout d'abord examiner si le statut organique antérieur peut expliquer la supériorité presque systématique des valeurs observées par rapport aux valeurs calculées.

Nous pensons que cela est peu plausible. En effet, un écart systématique tel que celui observé suppose un régime d'entretien organique antérieur plus riche sur toutes les situations et qui aurait échappé à toutes nos enquêtes.

Soient $\tau, \tau_{e}, \tau_{0}$ les teneurs en carbone ou matière organique respectivement au temps $t$, à l'équilibre, à l'origine du temps (fin de l'ancien régime d'entretien organique). Si l'intervalle d'intégration du bilan est suffisamment grand par rapport au pas de temps annuel (CHADOEUF, 1981), la cinétique d'évolution correspondant au modèle de HÉNIN-DUPUIS peut s'écrire :

$$
\tau=\tau_{\mathrm{e}}+\left(\tau_{0}-\tau_{\mathrm{e}}\right) \cdot \mathrm{e}^{-\mathrm{k}_{2 \mathrm{t}}}
$$

Si l'on pose :

$$
\tau-\tau_{\mathrm{e}}=\delta \tau
$$

et

$$
\tau_{0}-\tau_{\mathrm{e}}=\Delta \tau
$$

on a :

$$
\delta \tau=\Delta \tau \cdot e^{-k_{2} t}
$$

et réciproquement :

$$
\Delta \tau=\delta \tau \cdot \mathrm{e}^{\mathbf{k}_{2} \mathrm{t}}
$$

$\Delta \tau$ représente l'écart des teneurs d'équilibre entre l'ancien et le nouveau système de culture, $\delta \tau$ l'écart qui devrait être constaté, à une date $\mathrm{t}$ à partir du changement de système, entre la teneur observée et la teneur prévue $\tau_{\mathrm{e}}$. Le modèle étant supposé vrai et la condition d'équilibre fausse, $\delta \tau$ serait matérialisé sur les fig. 2 et 3 par l'écart d'un point expérimental à la $1^{\text {re }}$ bissectrice, l'abscisse étant fixée et l'écart mesuré sur l'ordonnée. Sous ces mêmes hypothèses, la relation (7) permet de calculer les couples de valeurs $(\Delta \tau$, t) qui rendent compte des valeurs $\delta \tau$ constatées en moyenne. Ainsi, si on prend $\mathrm{k}_{2}=0,014$ (valeur modale au sein de l'échantillon), on obtient les valeurs suivantes :

$$
\begin{aligned}
- \text { pour } \delta \tau= & 4,7 \% \text { (cas du carbone), } \\
\Delta \tau= & 7 \% \text { pour } \mathrm{t}=30 \text { ans et } \\
& 10 \% \text { pour } \mathrm{t}=50 \mathrm{ans} \\
- \text { pour } \delta \tau= & 3 \% \text { (matière organique), } \\
\Delta \tau= & 4,5 \% \text { pour } \mathrm{t}=30 \text { ans et } \\
& 6 \% \text { pour } \mathrm{t}=50 \text { ans. }
\end{aligned}
$$

Il en ressort que le biais systématique observé ne pourrait être expliqué, même si on retient provisoirement l'expression " matière organique », que par des diminutions assez importantes des régimes d'entretien organique du sol : il faut envisager des retournements de prairies permanentes et/ou une diminution importante de la disponibilité globale en fumier pour les terres labourables, intervenant dans tous les cas enquêtés et systématiquement oubliés par les agriculteurs. Ceci paraît d'autant plus improbable que la proportion de la SAU en surface toujours en herbe, qui a effectivement diminué dans la région après la $2^{\mathrm{e}}$ guerre mondiale, n'y a jamais dépassé 30 à 40 p. 100 et que, par ailleurs, l'effectif de bovins (donc la masse de fumier disponible pour les terres labourables) a plutôt augmenté depuis 1955. Il faut donc sans doute chercher ailleurs l'origine du biais systématique observé.

\section{Problème de la dispersion résiduelle}

Rien n'exclut une certaine hétérogénéité des statuts organiques antérieurs. Par rapport à la variabilité actuellement constatée, cette variabilité antérieure est a priori du même ordre de grandeur et en partie indépendante : cette indépendance n'est évidemment que partielle puisque le coefficient $k_{2}$ reste à peu près identique en chaque lieu ; par contre, les régimes d'apport sont sans doute beaucoup moins stables en chaque situation ; en particulier, les règles de distribution spatiale du fumier ont été modifiées du fait de la mécanisation.

En raison de la lenteur des évolutions, il est tout à fait plausible que cette hétérogénéité antérieure s'exprime encore actuellement sous forme d'écarts à la prévision. En effet, supposons que les agriculteurs aient « oublié » un changement de système de culture datant de 50 ans, on aurait alors, d'après la relation (6) et avec $\mathrm{k}_{2}=0,014$ :

$$
\delta \tau \simeq 0,5 \Delta \tau
$$

$\delta \tau$ apparaissant précisément comme un écart entre prévision et observation, et

$$
\text { variance }(\delta \tau) \simeq 0,25 \text {. variance }(\Delta \tau) \text {. }
$$

La variance de $\Delta \tau$ dépend de celles de $\tau_{\mathrm{o}}$, de $\tau_{\mathrm{e}}$ et de la covariance de ces 2 termes. Faute d'informations, nous ne pouvons pousser ce raisonnement plus loin. Cependant, on voit que la variance des écarts prévision-observation (qui correspond en fait à la 
variance résiduelle autour des relations 4 et 5 ) est fortement influencée par des variations de régime d'entretien organique même très anciennes. Il n'est donc guère surprenant que les coefficients de corrélation obtenus ( 0,7 environ) ne soient pas plus élevés.

\section{Discussion relative à l'estimation des apports}

\section{Problème du biais systématique}

Imputer ce biais à l'estimation des apports revient à mettre en cause une sous-estimation des coefficients $k_{1}$ et/ou une sous-estimation des masses d'apport.

L'hypothèse d'une sous-estimation des coefficients $\mathrm{k}_{1}$ est très tentante dans le cas de l'expression en carbone qui entraîne, avec le même jeu de coefficients $k_{1}$ que pour l'expression en matière organique, un décalage nettement plus important entre valeurs observées et valeurs prévues.

Il se trouve que nous disposons d'un moyen de contrôle partiel de cette hypothèse car il est possible d'individualiser le facteur prépondérant de variation des apports, le fumier de ferme, qui représente globalement plus de la moitié des masses de matière organique apportées au sol.

Dans la relation 2, on peut isoler la contribution du fumier en regroupant tous les apports autres, où prédominent très largement les résidus de culture, soit $\mathrm{k}_{1 \mathrm{c}} \mathrm{m}_{\mathrm{c}}$. On peut ainsi écrire :

$$
\tau_{\mathrm{e}} \cdot \mathrm{k}_{2} \cdot \mathrm{M}=\mathrm{k}_{1 \mathrm{c}} \mathrm{m}_{\mathrm{c}}+\mathrm{k}_{1 \mathrm{~F}} \mathrm{~m}_{\mathrm{F}} .
$$

Le terme de gauche de cette relation (soit $\phi$ ) est le flux moyen annuel de minéralisation à l'équilibre. Il peut être calculé sur chaque situation en supposant que $\tau_{\mathrm{e}} \simeq \tau$ et en utilisant les valeurs $\mathrm{k}_{2}$ et $\mathbf{M}$ appropriées. On a, par ailleurs, estimé les apports de fumier, soit $\mathrm{m}_{\mathrm{F}}$. Par conséquent, si l'on dispose d'un nombre important de parcelles connues et d'une gamme suffisante de variation sur $m_{F}$, on doit pouvoir mettre en évidence une relation statistique de forme :

$\Phi=\alpha \mathrm{m}_{\mathrm{F}}+\beta$ telle que $\alpha \simeq \mathrm{k}_{1 \mathrm{~F}}$ et $\beta \simeq \mathrm{k}_{1 \mathrm{c}} \mathrm{m}_{\mathrm{c}}$.

Cette relation ne peut apparaître que $s i \mathrm{k}_{1 \mathrm{c}} \mathrm{m}_{\mathrm{c}}$ est peu variable. Nous avons donc découpé l'ensemble des résultats selon 3 classes de valeurs de $k_{1 c} m_{c}$ estimées. Les résultats concernant l'expression en carbone sont donnés dans le tableau 2. Nous obtenons ainsi une

TABLEAU 2

Estimation du coefficient $k_{l}$ du fumier d'après les régressions.

$$
\Phi=\alpha \mathrm{m}_{\mathrm{F}}+\beta .
$$

Estimation of coefficient $k_{\perp}$ for farmyard manure from regressions.

$$
\Phi=\alpha \mathrm{m}_{\mathrm{F}}+\beta .
$$

\begin{tabular}{lcccc}
\hline \hline $\begin{array}{l}\text { Classes de } k_{1 c} m_{c} \\
\text { calculées }(\mathrm{kg} / \mathrm{ha})\end{array}$ & $\begin{array}{c}\text { Estimation } \\
\text { de } \alpha\end{array}$ & $\begin{array}{c}\text { Estimation } \\
\operatorname{de} \beta\end{array}$ & $\mathbf{r}$ & Effectif \\
\hline 100 à 175 & 0,352 & 423 & $0,63^{* *}$ & 23 \\
175 à 200 & 0,335 & 418 & $0,81^{* *}$ & 19 \\
200 à 225 & 0,324 & 523 & $0,69^{* *}$ & 24 \\
\hline \hline
\end{tabular}

estimation remarquablement stable $\mathrm{du}$ coefficient $\mathrm{k}_{1}$ correspondant au carbone, soit en moyenne pondérée $\mathrm{k}_{1 \mathrm{~F}}=0,34$. Cette valeur s'avère :

- très proche de celle déterminée récemment par DELAS \& MOLOT (1983), soit 0,32 ;

- peu différente également de celle choisie pour les calculs $(0,30)$, alors que si on effectue les calculs en matière organique, on obtient une valeur moyenne estimée de 0,21 , plutôt inférieure.

On peut donc en conclure que, au moins pour ce qui concerne le fumier de ferme, c'est-à-dire globalement pour plus de la moitié des apports, il n'y a pas eu sous-estimation importante des coefficients $k_{1}$ en cas d'expression des résultats en carbone.

Notre évaluation du terme $k_{1} m$ en carbone a donc été vraisemblablement plutôt plus représentative de la réalité que notre évaluation en matière organique. C'est bien ce que traduit la comparaison des pentes des régressions correspondant aux relations 4 et 5 : le coefficient est proche de 1 dans le cas de l'expression en carbone, ce qui signifie que, dans ce cas, le modèle de HÉNIN-DUPUIS a correctement prévu, sinon les teneurs d'équilibre (problème du biais systématique), en tout cas le classement des situations observées. Nous avons d'ailleurs pu nous assurer (KÉLI, 1984) que cette configuration restait stable si on considérait différentes classes de valeurs de $\mathrm{k}_{2}$.

Reste alors l'hypothèse d'une sous-estimation des masses d'apport (terme m). Celle-ci ne peut être générale que si elle concerne les racines, seul apport strictement commun à toutes les parcelles. Or, on a retenu (Annexe 1) des valeurs du rapport biomasse aérienne/ biomasse racinaire relativement faibles par rapport aux valeurs couramment admises. En comparant les valeurs estimées de $\beta$ et de $k_{1 c} m_{c}$ du tableau 2 , on voit que le biais porte sur environ $300 \mathrm{~kg}$ de carbone $\mathrm{du}$ sol, soit avec un rendement de $15 \mathrm{p} .100,2 \mathrm{t}$ d'apport brut de carbone au sol représentant environ $4 \mathrm{t}$ de matière sèche. Une sous-estimation d'une telle ampleur est peu plausible. Certes, des auteurs comme SAUERBECK \& JOHNEN (1977) ont montré que la décomposition des racines en cours de végétation concerne des quantités beaucoup plus importantes que celle des racines à la récolte. Mais rien ne permet de considérer qu'à l'échelle d'une année, on doive affecter la totalité de ces apports d'un coefficient $k_{1}$ de 0,15 , coefficient isohumique « apparent » retenu ici pour les racines; en effet la décomposition en cours de végétation concerne surtout un matériau jeune probablement pauvre en lignine.

\section{Problème de la dispersion résiduelle}

Nous avons noté (tabl. 1) que la dispersion résiduelle était liée, pour une part modeste mais significative, à l'estimation des apports : aux plus faibles estimations correspondent, en moyenne, des valeurs observées situées au-dessus de la droite de régression. Ceci pourrait s'expliquer par une sous-estimation de la contribution des résidus de culture (autres que les racines) par rapport à celle du fumier.

Enfin, nous ne pensons pas que l'imprécision dans la connaissance des apports joue à elle seule un rôle décisif vis-à-vis de la dispersion des résultats autour des droites de régressions. En effet, en isolant sur la 
figure 3 les 24 points pour lesquels les apports sont connus avec une précision nettement supérieure (durée des successions de culture connues supérieure à 15 ans, rendements connus au niveau de la parcelle), on obtient une configuration aussi dispersée que pour l'ensemble $(r=0,686)$.

\section{E. Discussion relative à l'estimation des pertes}

Par défaut, les discussions précédentes suggèrent que c'est à cette partie du calcul qu'il faut imputer la plus grande responsabilité quant au biais systématique observé. Il faudrait, pour cela, admettre que les valeurs de $\mathrm{k}_{2}$ ont été surestimées d'environ 30 p. 100 et sont centrées sur une valeur d'environ 0,01 (au lieu de 0,014).

Une telle valeur, quoique nettement plus faible que les références couramment citées dans le cas de sols limono-argileux, n'est pas pour autant aberrante. Sur les essais de longue durée du champ de DEHÉRAIN (situés à environ $100 \mathrm{~km}$ au sud de la zone étudiée, sur un sol de texture limono-argileuse) MOREL et al. (1956), MOREL (1968), ont déterminé des valeurs de $k_{2}$ nettement inférieures à 0,01 (jusqu'à 0,006 ) pour des parcelles à régime de restitution nulle.

Ceci nous amène à un réexamen critique de la procédure d'estimation utilisée pour $\mathrm{k}_{2}$ (relation 3 ).

Remarquons tout d'abord que la forme de la relation 3 est compatible avec la liaison d'allure linéaire fréquemment observée entre teneur en matière organique et teneur en argile. Il suffit pour le vérifier de remplacer, dans la relation 1 , le terme $k_{2}$ par son expression donnée dans la relation 3 en supposant la teneur en calcaire constante. Pour simplifier on peut écrire :

$$
\begin{gathered}
\mathrm{k}_{2}=\frac{\mathrm{u}}{\mathrm{A}+\mathrm{v}}(\mathrm{u}, \mathrm{v} \text { constantes }) \\
\tau_{\mathrm{c}}=\frac{(\mathrm{A}+\mathrm{v}) \cdot \mathrm{k}_{1} \cdot \mathrm{m}}{\mathrm{u} \cdot \mathrm{M}} .
\end{gathered}
$$

On est donc amené à remettre en question les valeurs numériques données aux coefficients $u$ et $v$.

Diminuer le coefficient $u$ reviendrait à diminuer l'écart entre les valeurs prévues et observées, mais aussi à augmenter l'écart de teneur prévu entre des situations ne différant que par la teneur en argile. Ceci aurait pour conséquence une diminution de la pente générale de la régression valeurs observées $=\mathrm{f}$ (valeurs prévues), et donc un moins bon ajustement des résultats puisque cette pente est d'ores et déjà inférieure à 1 . C'est donc plutôt le coefficient $v$, qui n'affecte pas cette pente, qu'il conviendrait de remettre en cause.

Au-delà de ces considérations strictement empiriques, c'est peut-être le caractère simpliste du schéma d'évolution des matières organiques sur lequel repose le modèle de HÉNIN-DUPUIS qui est à mettre en cause.

Il est en effet bien connu que l'ensemble des matières organiques du sol regroupe des fractions dont les vitesses d'évolution sont très différentes, une part non négligeable étant redevable d'un coefficient de minéralisation beaucoup plus faible que les valeurs de $\mathrm{k}_{2}$ couramment admises (JENKINSON \& RAYNER, 1977). Il est donc possible que des régimes d'entretien organique restreints aboutissent à des teneurs plus élevées que prévu, correspondant à des formes organiques plus résistantes.

\section{CONCLUSION}

La confrontation réalisée montre que le modèle de HÉNIN-DUPUIS et l'ensemble des références numériques qui lui sont associées permet de classer, de manière globalement satisfaisante, les situations culturales et les régimes d'entretien organique qui leur sont appliqués. Il est donc possible de prévoir le sens de variation d'une teneur lorsque le système de culture appliqué à une parcelle est modifié. Ceci est en soi une réponse positive importante pour les prescripteurs qui ont à prendre en compte l'évolution de la fertilité dans leurs conseils aux agriculteurs.

Il n'est pas surprenant, compte tenu des procédures d'enquête auxquelles nous avons recouru, que subsistent des écarts résiduels assez importants autour de la régression entre valeurs prévues et valeurs observées. Une certaine hétérogénéité des statuts organiques antérieurs, jointe à la lenteur de passage d'un équilibre à un autre, suffisent à en rendre compte.

En revanche, l'observation d'un écart systématique entre les teneurs observées et les teneurs prévues (le plus souvent inférieures) conduit à réexaminer les hypothèses et estimations sur lesquelles s'est appuyée la démarche. Après discussion, on est amené à soupçonner, en premier lieu, une surestimation systématique de la minéralisation, ce qui n'exclut pas que d'autres causes aient pu contribuer dans tel ou tel cas à faire sous-estimer les teneurs d'équilibre.

Pour faciliter l'utilisation du modèle de HÉNINDUPUIS en tant qu'instrument de diagnostic régional sur les systèmes de culture, il faudrait non seulement améliorer l'estimation de $\mathrm{k}_{2}$, mais aussi que les tableaux de références numériques concernant $k_{1}$ soient clairement assortis du mode d'expression retenu, matière organique ou carbone, voire réactualisés en tenant compte de ce choix. Une contribution de notre travail à cette entreprise est la confirmation de la valeur moyenne du coefficient $k_{1}$ du fumier de stabulation, récemment obtenue par voie expérimentale par DELAS \& MOLOT (1983) et que nous retrouvons par une approche tout à fait différente.

La réalisation d'enquêtes du même type dans d'autres régions permettrait de vérifier si l'on retrouve ou non cette sous-estimation des valeurs calculées par rapport aux valeurs observées. Ceci pourrait déboucher sur un réajustement des procédures d'estimation du coefficient de minéralisation, voire même sur une critique constructive du modèle monocompartimental. 


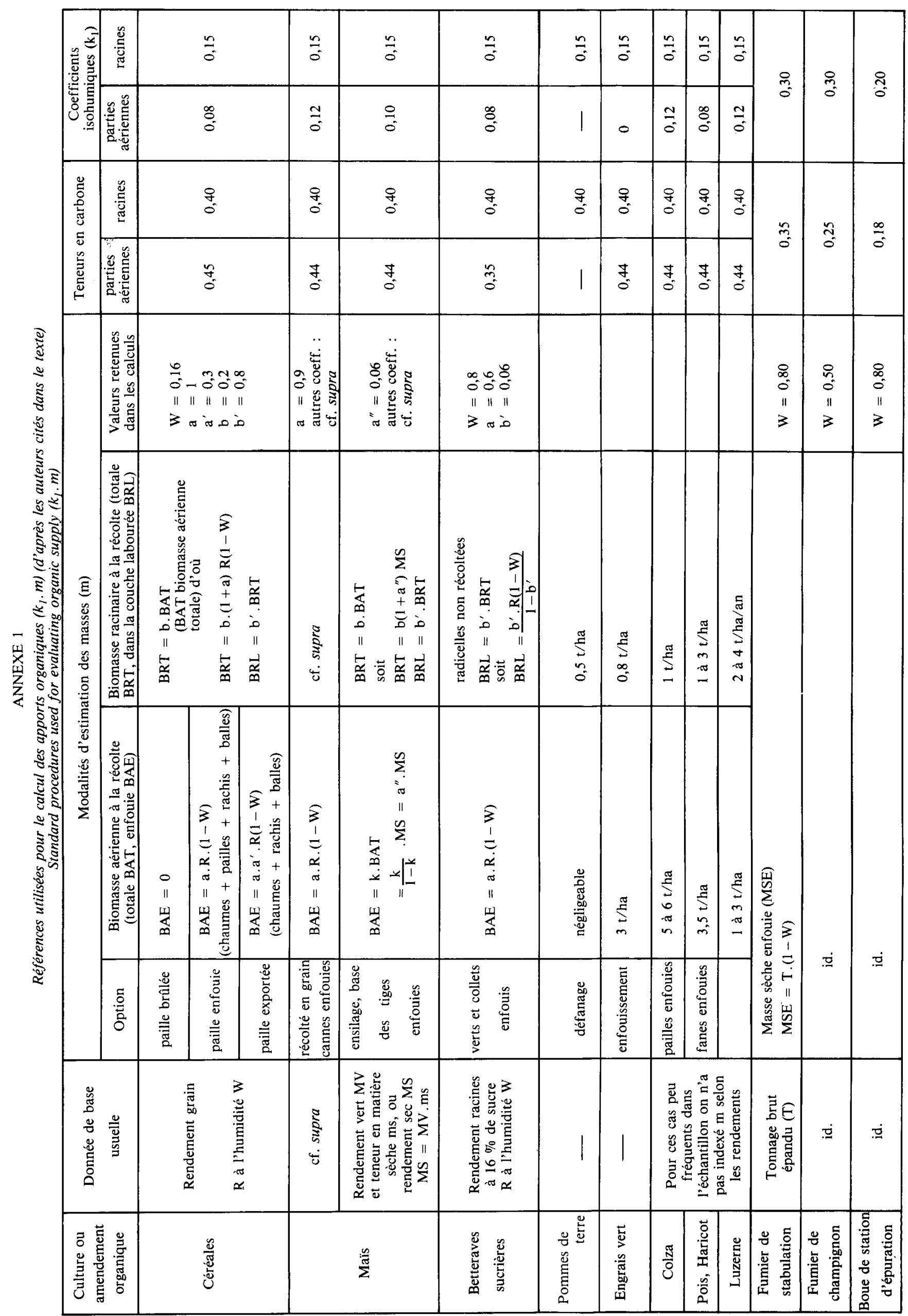




\section{RÉFÉRENCES BIBLIOGRAPHIQUES}

Aubry C., Capillon A., Servettaz L., 1986. Diversité des systemes de production du Noyonnais et leur sensibilité au milieu. A paraître dans "Fertilité et système de production en région de grande culture ». DGRST-ECAR-INAPG.

Begon J. C., Mori A., Hardy Y. R., 1976. Les sols de l'Oise -Notice de la carte pédologique au $1 / 100000$, I.N.R.A., Service de la Carte.

Boiffin J., Fleury A., 1974. Quelques conséquences agronomiques du retournement des prairies permanentes. Ann. agron., 25 (4), 555 573.

Broadbent F. E., 1953, cité in F. E. Al.lison, 1973. Soil organic matter and its role in crop production - Elsevier Scientific P.C.. Amsterdam, London, New York, 637 p.

Chadouf J., 1981. Modélisation de l'évolution des matières organiques du sol; estimation des paramètres et simulation. Mémoire DEA, Univ., Paris XI, 36 p. + annexes.

Delas J., Molot C., 1983. Effet de divers amendements organiques sur les rendements du maïs et de la pomme de terre cultivés en sol sableux. Ann. agron., 3 (1), 19-26.

Delphin J. E., Conesa A. Ph., 1979. Evolution de la matière organique du sol d'un essai rotation, irrigation, restitution des pailles, dans la plaine de la Hardt. II. Bilan humique. Ann. agron., 30 (2), 179-189.

Delphin J. E., Tiquet J. L., 1986. Appréciation par l'analyse de terre de l'aptitude à la minéralisation de sols de la plaine d'Alsace. A paraître dans "Fertilité et système de production en région de grande culture ». DGRST-ECAR-INAPG.

Guérif J., 1982. La matière organique du sol et son évolution. Bull. Tech. Inform. Min. Agric., 370/372, 443-450.

Guérif J., Faure A., 1979. Rôle de la matière organique sur le comportement des sols au compactage. Ann. agron., 30 (5), 387-399.

Hénin S., Dupuis M., 1945. Essai de bilan de la matière organique des sols. Ann. agron., 15 (1), 161-172.

Hénin S., Turc L., 1949. Essai de fractionnement des matières organiques du sol. C. R. Acad. Agric. Fr., 35 (1), 41-43.

Hénin S., Monnier G., Turc L., 1959. Un aspect de la dynamique des matières organiques du sol. C. R. Acad. Sci., Paris, 248, 138-141.

Jacquin F., 1963. Notions récentes concernant le bilan humiąule d'un sol. Bull. Assoc. Fr. Etude Sol., 11, 442-499.

Jenkinson D. S., Rayner J. H., 1977. The turnover of soil organic matter in some of the Rothamsted classical experiments. Soil Sci., 123 (5), 298-305.

Kéli J., 1984. Système de culture, système de production et statut organique des sols : essai de diagnostic sur les pratiques d'entretien organique dans une petite région agricole. Thèse Doct. Ing., INAPG, $208 \mathrm{p}$.
Monnier G., 1965. Action des matières organiques sur la stabilité structurale des sols. Thèse Doct. Ing., Fac. Sciences, Paris, $140 \mathrm{p}$.

Monnier G., 1966. Les restitutions organiques. Leur action sur le sol. Bull. C.E.T.A., fév. 1966, 5-14.

Monnier G., Turc L., Jeanson-Luusinang V., 1962. Une méthode de fractionnement densimétrique par centrifugation des matières organiques du sol. Ann. agron., 13 (1), 55-63.

Morel R., 1968. Evolution de l'azote et du carbone organique du sol au cours d'une expérience de longue durée. Ann. agron., 19 (2), 153-174.

Morel R., Richer A., Masson P., 1956. Etude expérimentale de la variation du taux de matière organique dans le champ d'expérience de la station agronomique de Grignon. Ann. agron., 7 (2), 183-204.

Morizet J., Millet R., 1971. Etude de la mobilité de l'eau non saturante dans des colonnes de sol soumises à l'évaporation. Influence de la texture et de la teneur en matière organique. Ann. agron., 22 (2), 197-211.

Muller J., 1966. Observation sur les effets à long terme des fumures organiques et minérales sous climat méditerranéen. II. Action sur le bilan de l'azote total du sol. Ann. agron., 17 (1), 21-26.

Muller J., 1982. Matière organique : à quel niveau la maintenir ? Cultivar, janvier 1982, 60-66.

Myers H. E., 1937, cité par C. E. MiLlar, 1955. Soil fertility. J. Wiley \& Sons, New York, $436 \mathrm{p}$.

Osty P. L., 197। a. Essai de quantification de l'aptitude à la fissuration de terres très argileuses par délitement dans l'alcool éthylique. Recherche du rôle de différents facteurs. C. R. Acad. Agric. Fr., 47 (1), 75-87.

Osty P. I... 1971b. Influence des constituants du sol sur son humidité à pH3. Ann. agron., 22 (4), 451-476.

Rémy J. C., 1971. Influence de la constitution physique des sols sur leur comportement mécanique : signification des limites d'Atterberg en matière de travail du sol. Ann. agron., 22 (3), 267-290.

Rémy J. C., Marin-Laflèche A., 1974. L'analyse de terre ; réalisation d'un programme d'interprétation automatique. Ann. agron., 25 (4), 607-632.

Rémy J. C., Marin-Laflèche A., 1976. L'entretien organique des terres. Coût d'une politique de l'humus. Entreprises agricoles, novembre 1976, 63-67.

Rémy J. C., Orsini L., Marin-Laflèche A., 1975. L'intérêt agronomique de quelques sous-produits utilisables en grande culture. $\mathrm{Hau}$ tes Etudes betteravières et agricoles, 31, 17-29.

Ruellan A., Deletang J., 1967. Les phénomènes d'échange de cations et d'anions dans les sols. Initiations-documentations techniques $\mathrm{n}^{\circ}$ 5, ORSTOM Paris, $123 \mathrm{p}$.

Sauerberck D. R., Johnen B. G., 1977. Root formation and decomposition during plant growth, in "Soil organic matter studies", vol. 1, I.A.E.A., Vienna, 424 p. 